-Mini Review-

\title{
Localization and Function of Cyclin B1 and Cyclin B2 during Porcine Oocyte Maturation
}

\author{
Takao Kuroda ${ }^{1}$ and Kunihiko Naito ${ }^{1 *}$ \\ ${ }^{1}$ Department of Applied Genetics, Graduate School of Agriculture and Life Science, University of \\ Tokyo, Bunkyo-ku, Tokyo 113-8657, Japan
}

Oocyte maturation is regulated by maturation/Mphase promoting factor (MPF), a crucial M-phase regulating enzyme composed of a catalytic subunit, $\mathrm{p} 34^{\mathrm{cdc} 2}$, and a regulatory subunit, cyclin B. The amount of p34 ${ }^{\mathrm{cdc} 2}$ is almost constant during oocyte maturation, and the amount of cyclin $B$ is the principal factor determining MPF activity [1]. The presence of two types of cyclin B, cyclin B1 and cyclin B2, has been shown in vertebrates. In human cells, cyclin B1 can cause chromosome condensation, reorganization of the microtubules, and disassembly of the nuclear lamina and of the Golgi apparatus, whereas the role of cyclin B2 is restricted only to disassembly of the Golgi apparatus [2, 3]. In maturing oocytes, differences between cyclin B1 and cyclin B2 functions have been reported in the first meiotic spindle formation and the second metaphase arrest in frog and mouse oocytes, respectively [4-6]. In our laboratory, we have studied cyclin $B$ functions during porcine oocyte maturation for the past several years. The present review describes our recent observations with regard to protein levels, intracellular localizations and roles of cyclin B. We focus here on the differences between cyclin B1 and cyclin B2.

\section{Protein Levels of Cyclin B1 and Cyclin B2 during Meiotic Maturation of Porcine Oocytes}

We used immunoblotting to examine the protein levels of cyclin B1 and cyclin B2, looking at the density of the bands at $62 \mathrm{kDa}$ and $45 \mathrm{kDa}$, respectively. In immature porcine oocytes, the presence of a small amount of pre-MPF, a hyperphosphorylated inactiveMPF, has been suggested [7-9], but the relative amount

Received: August 6, 2003

Accepted: September 19, 2003

*To whom correspondence should be addressed.

e-mail: aknaito@mail.ecc.u-tokyo.ac.jp and the kind of cyclin B comprising the pre-MPF have never been clarified. Our resent experiment with 200 porcine oocytes revealed that cyclin B1 was absent from the immature oocytes, whereas the amount of cyclin B2 in immature oocytes was between 1/20 and 1/ 40 of that in the first metaphase oocytes (the intensity of the cyclin B2 band of 200 non-cultured oocytes was between 5 and 10 oocytes in oocytes cultured for 24-h: Fig. 1A).

Cyclin B1 and cyclin B2 were detected just before germinal vesicle breakdown (GVBD), which was induced in most of the oocytes at 20-24 $\mathrm{h}$ in the present culture conditions [10-12]. Thereafter, cyclin B1 gradually increased, with a transient decrease at the first polar body extrusion, and reached its peak level at the second meiotic metaphase ( $48 \mathrm{~h}$ of culturing). In contrast, cyclin B2 reached its peak level at the first metaphase, then decreased abruptly in correlation with the first polar body extrusion and maintained a low level during the second meiosis (Fig. 1B). In Xenopus oocytes, the degradation of cyclin B2 at the first meiosis/ second meiosis transition and the requirement of cyclin B1 synthesis for the second meiosis induction have been reported [13]. Except for the cyclin $B$ levels in immature oocytes, the cyclin B fluctuation patterns in porcine oocytes agreed well with those reported in Xenopus and mouse oocytes [14-16]. These results suggest that cyclin B2 works during the first meiosis, whereas cyclin B1 works mainly during the second meiosis.

\section{Intracellular Localization of Cyclin B1 and Cyclin B2 during Porcine Oocyte Maturation}

Intracellular localization of cyclin B has been examined by immunocytochemical methods with antibodies specific for each cyclin B. In agreement with the protein levels described above, no cyclin B1 signal 

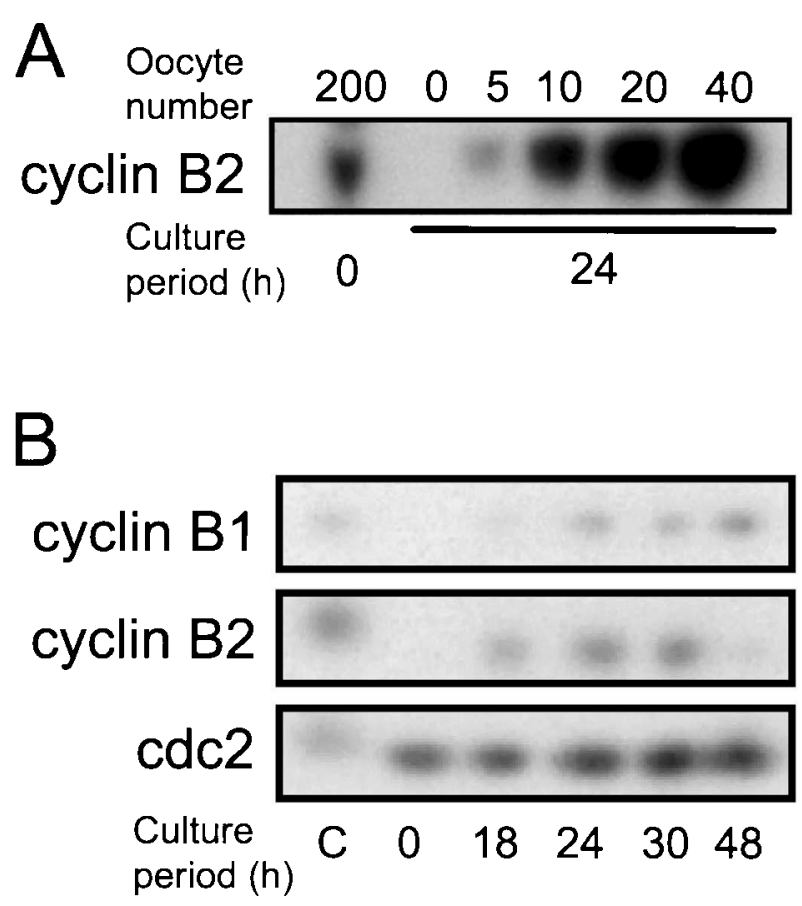

Fig. 1. The amounts of cyclin B1 and cyclin B2 during meiotic maturation of porcine oocytes. (A) The amount of cyclin B2 in immature porcine oocytes. The 200 noncultured oocytes and an increasing number of $24 \mathrm{~h}$ cultured oocytes were used for immunoblotting. (B) Protein levels of cyclin B1 (upper panel), cyclin B2 (middle panel) and cdc2 (lower panel). Ten oocytes were subjected to the immunoblotting at each of the culture periods indicated. Different parts of the same membrane were used for the detection of each protein.

and only a faint cyclin B2 signal were observed in immature porcine oocytes. Cyclin B2 localization was unclear at $0 \mathrm{~h}$ of culturing, but it probably occurred on the nuclear membrane, and then the cyclin B2 signal had increased in the whole germinal vesicle (GV) at 18 $\mathrm{h}$ when the MPF activity of the oocytes started to increase (Fig. 2A). In cultured cell lines, cyclin B is present in the cytoplasm during the inter-phase, and it translocates into the nucleus after the activation of MPF at the start of the M-phase [17-19]. In starfish oocytes, cytoplasmic cyclin B in immature oocytes translocates into GV just before GVBD [20]. Therefore, the accumulation of active MPF in the nucleus before the start of the M-phase might be common to all cell types.

After GVBD, the cyclin B1 signal increased in the whole cytoplasm, with no localization detected during the first meiosis, whereas clear localization on both poles of the spindle was observed at the second meiotic metaphase (Fig. 2B) [21, 22]. This suggests the role of cyclin B1 in spindle formation during the second meiosis. The localization of cyclin B1 on the spindle poles has also been reported in cultured human cells [4, 19]. On the other hand, cyclin B2 co-localized with tubulin on the whole spindle during both the first and second meiosis, but its signal was much decreased in the second meiosis, suggesting the involvement of cyclin B2 in the first meiotic spindle formation (Fig. 2C). Indeed, the requirement of cyclin B2, but not cyclin B1, for the first meiotic spindle formation has been reported in Xenopus and Rana japonica oocytes [5, 23]. In porcine oocytes, however, cyclin B2 might not always be necessary for the first meiotic spindle formation, as described below. The localization of cyclin B on chromosomes has been reported previously in porcine oocytes [24]. We revealed, however, that cyclin B1 did not localize on somatic nuclei injected into matured porcine oocytes unless a spindle formed around the nucleus [21]. This result shows that porcine cyclin B1 does not bind with chromosomes directly. The cyclin B binding with tubulin has also been shown in biochemical experiments [20].

It should be emphasized that the immunocytochemical results in previous reports $[5,23$, $25,26]$ showing cyclin B localization on the spindle did not exclude its presence in the cytoplasm during the $\mathrm{M}$ phase. Weak signals were always detected in the cytoplasm on immunostaining with cyclin B-specific antibodies, indicating partiality to their localization on the spindle. Our experiments in removing spindles from mature porcine oocytes indicated very little cyclin B localization on spindles and showed that the majority of cyclin B remains in the cytoplasm [22]. In somatic cells, cyclin B2 localization on Golgi apparatus has been reported [2]. It remains to be determined whether cyclin B2 localizes on Golgi apparatus in porcine oocytes.

\section{Roles of Cyclin B1 and Cyclin B2 during Meiotic Maturation of Porcine Oocytes}

To analyze the roles of cyclin B1 and cyclin B2 during porcine oocyte maturation, we prepared their antisense RNAs in vitro [27] and injected one or the other or both into the cytoplasm of immature oocytes. Each antisense RNA inhibited objective cyclin B synthesis specifically without affecting the other cyclin $B$ synthesis [27].

When cyclin B1 antisense RNA was injected into oocytes, the injected oocytes underwent GVBD at the normal rate and over the normal time course, reaching a morphologically normal first meiotic metaphase. The 


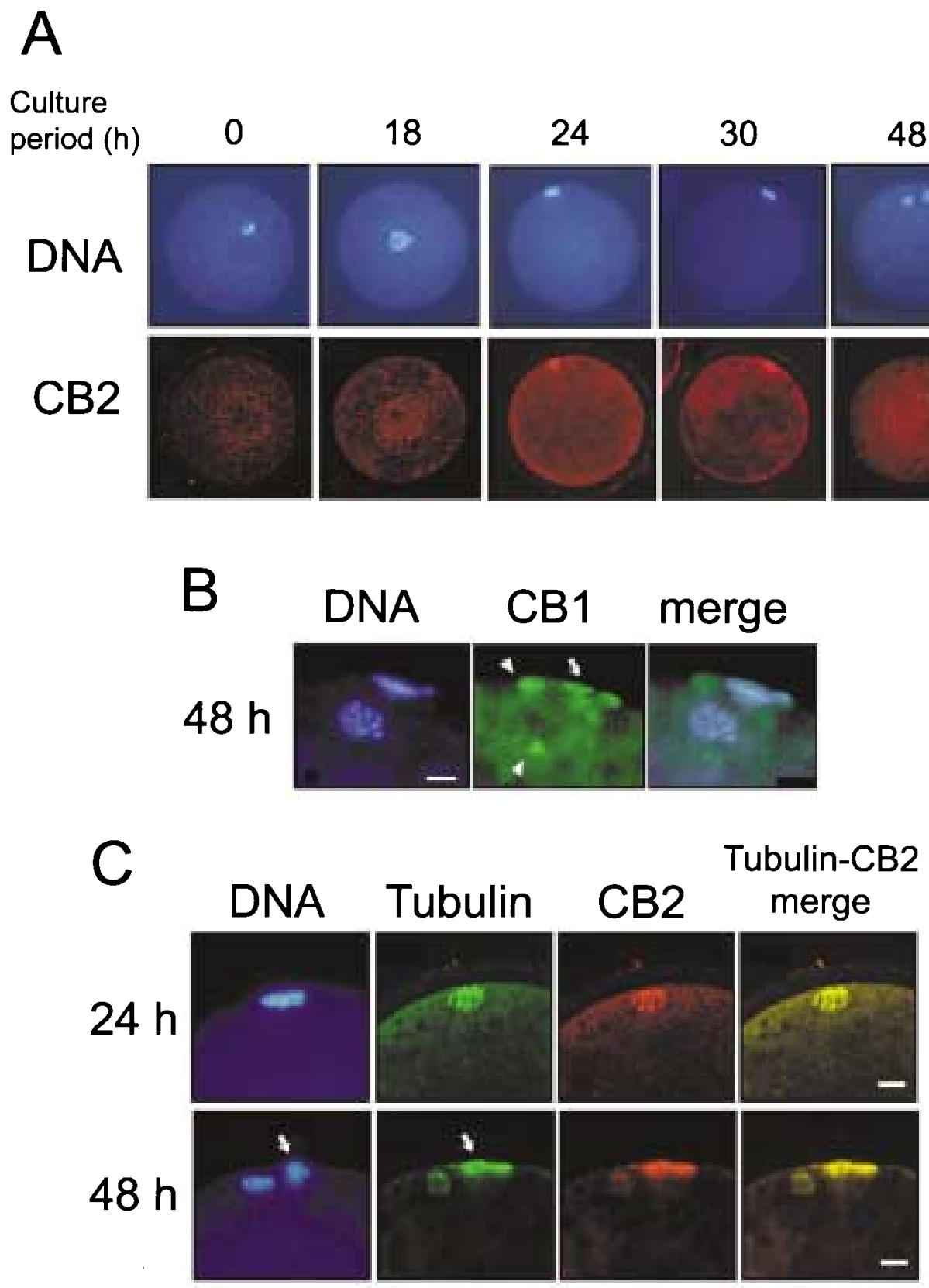

Fig. 2. Localization of cyclin $\mathrm{B} 1$ and cyclin $\mathrm{B} 2$ during meiotic maturation of porcine oocytes. (A) Oocytes were stained in the culture period indicated with Hoechst 33342 (DNA) and anticyclin B2 antibody (CB2). Each pair of photographs was taken of the same oocytes. (B) A mature oocyte was stained with Hoechst 33342 (DNA) and anti-cyclin B1 antibody (CB1). The first polar body and the spindle poles are indicated by an arrow and arrowheads, respectively. (C) Oocytes cultured for $24 \mathrm{~h}$ or $48 \mathrm{~h}$ were stained with Hoechst 33342 (DNA), anti-tubulin antibody (Tubulin) and anti-cyclin B2 antibody (CB2). The anti-cyclin B2 antibody was visualized with TRITC (red), and anti-tubulin and anti-cyclin B1 antibodies were visualized with FITC (green). The first polar bodies are indicated by arrows. Scale bars indicate $10 \mu \mathrm{m}$. 


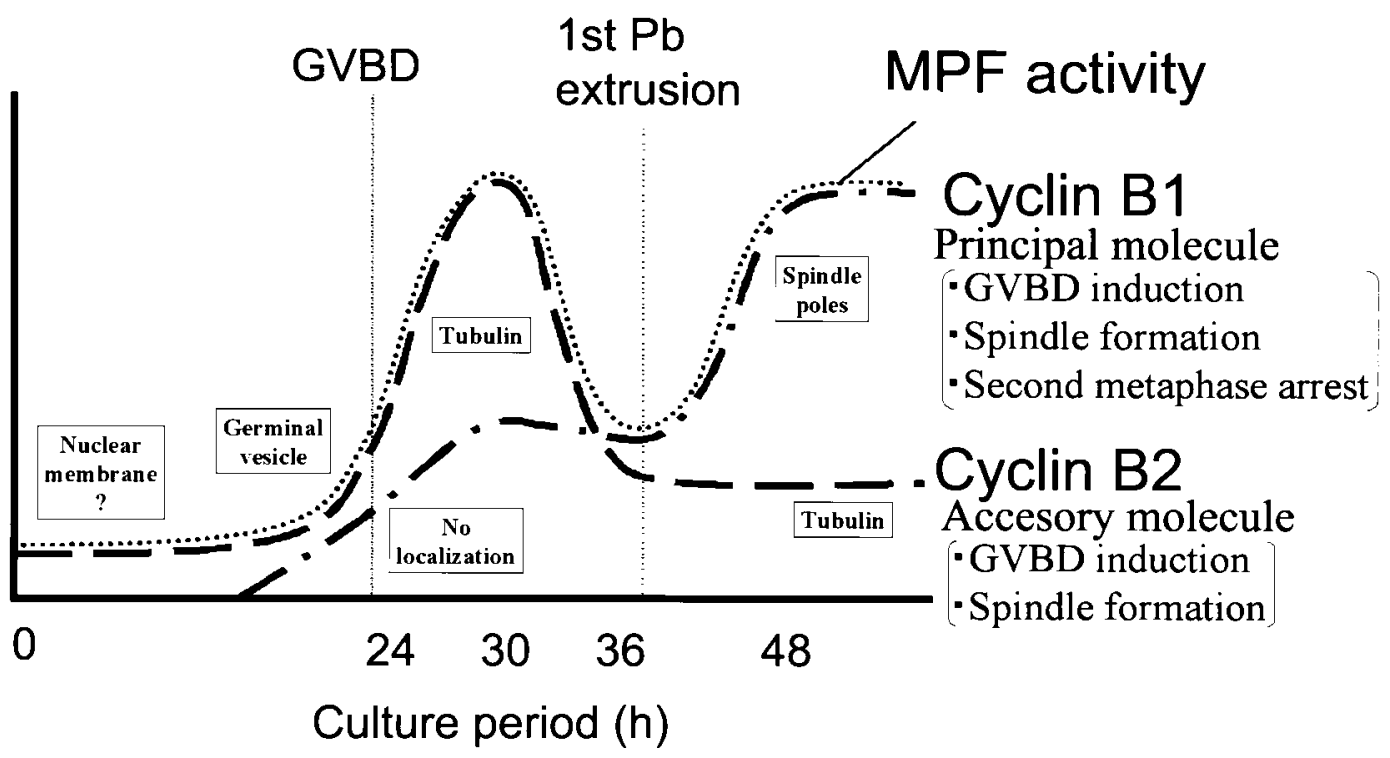

Fig. 3. Schematic diagram of the protein levels (line drawings), the intracellular localizations (boxes) and the roles (parentheses) of cyclin B1 and cyclin B2 during porcine oocyte maturation.

oocytes extruded first polar bodies normally, but they could not be arrested at the second meiotic metaphase, and almost all of the oocytes formed a pronucleus [27]. This result agrees well with the abovementioned suggestion that cyclin B2 works during the first meiosis, whereas cyclin B1 works mainly during the second meiosis. In mouse oocytes, the formation of a pronucleus was reported when cyclin B1, but not cyclin B2, synthesis was inhibited [6], indicating that the second meiosis is regulated mainly by cyclin B1 in mouse oocytes as well.

When cyclin B2 antisense RNA was injected into oocytes, GVBD induction in the oocytes was almost normal, in spite of the suggested importance of cyclin B2 during the first meiosis. Furthermore, no obvious morphological abnormality was detected in the first meiotic metaphase or the first polar body extrusion. The only detected effect of cyclin B2 suppression was the acceleration of first polar body extrusion, suggesting the maintenance effect of cyclin B2 for the first metaphase [27]. It has been reported that cyclin B2deficient mice were normal, in stark contrast to the morbidity of cyclin B1-deficient mice [28]. The wide range of cyclin B1 roles and the restricted roles of cyclin B2 have also been reported in cultured human cells [3]. Therefore, it seems likely that most of the roles of cyclin B2 could be compensated for by cyclin B1 in mammalian cells, including porcine oocytes. These results suggest that cyclin $\mathrm{B} 1$ is the principal molecule for M-phase regulation in mammalian cells and that cyclin B2 plays only an accessory role.

Finally, both cyclin B1 and cyclin B2 antisense RNAs were injected into porcine immature oocytes so that we could examine the requirement of cyclin $B$ synthesis for GVBD. The induction of GVBD in the injected oocytes was significantly suppressed but occurred slowly. Their MPF activity was significantly lower than that in control oocytes but tended to increase gradually [27]. This shows that the small amount of pre-MPF present in the immature oocytes was activated without de novo cyclin $B$ synthesis and could induce GVBD, although the timecourse was clearly slow. It could be concluded that, in porcine oocytes, cyclin B synthesis was not necessary for GVBD induction per se, but synthesis of one cyclin $B$, either B1 or B2, was necessary for GVBD induction in a normal time course. This situation is the intermediate between those oocytes whose GVBD was not affected by the inhibition of cyclin B synthesis, such as in Xenopus and mouse [6, 13, 29], and those oocytes whose GVBD was completely prevented by the inhibition of cyclin B synthesis, such as Rana japonica [30].

\section{Conclusion}

The protein levels, intracellular localizations and roles of cyclin B1 and cyclin B2 during porcine oocyte maturation are summarized in Fig. 3. Porcine immature oocytes had only cyclin B2, at a level between $1 / 20$ and 
$1 / 40$ of that in first metaphase oocytes. Both cyclin B syntheses were started around GVBD; cyclin B1 and cyclin B2 peaked at the second metaphase and first metaphase, respectively. Only cyclin B2 localizes into GV just before GVBD. After GVBD, although cyclin B1 and cyclin B2 localize on spindle poles and tubulin, respectively, most of the cyclin $B$ remains in the cytoplasm. The inhibition of cyclin B1 synthesis has no effect on the first meiosis but causes failure of the second meiotic metaphase arrest, and the inhibition of cyclin B2 synthesis has almost no effect on porcine oocyte maturation, suggesting that the principal molecule for $\mathrm{M}$-phase regulation is cyclin $\mathrm{B} 1$ and that cyclin B2 is an accessory molecule. Cyclin B synthesis is not required for GVBD induction in porcine oocytes, but at least one cyclin B synthesis is necessary for GVBD induction in a normal time course.

\section{Acknowledgment}

We are grateful to our colleagues Koji Sugiura, Naoki Iwamori, Satoshi Ohashi, Seitaro Goto, and Ikuko Takakura for their critical contributions to this manuscript. I would specially like to thank Dr. Hidaki Tojo for his directions. The work in our laboratory was supported by a Grant-in-Aid for Scientific Research (14360173) to K. N. from the Japan Society for the Promotion of Science.

\section{References}

1) Nurse, P. (1990): Universal control mechanism regulating onset of M-phase. Nature, 344, 503-508.

2) Jackman, M., Firth, M. and Pines, J. (1995): Human cyclins B1 and B2 are localized to strikingly different structures: B1 to microtubules, B2 primarily to the Golgi apparatus. EMBO J., 14, 1646-1654.

3) Draviam, V.M., Orrechia, S., Lowe, M., Pardi, R. and Pines, J. (2001): The localization of human cyclin B1 and B2 determines CDK1 substrate specificity and neither enzyme requires MEK to disassemble the Golgi apparatus. J. Cell Biol., 152, 945-958.

4) Clute, P. and Pines, J. (1999): Temporal and spatial control of cyclin B1 destruction in metaphase. Nat. Cell Biol., 1, $82-87$.

5) Yoshitome, S., Furuno, N. and Sagata, N. (1998): Overexpression of the cytoplasmic retention signal region of cyclin B2, but not of cyclin B1, inhibits bipolar spindle formation in Xenopus oocytes. Biol. Cell, 90, 509-518.

6) Ledan, E., Polanski, Z., Terret, M.E. and Maro, B. (2001): Meiotic maturation of the mouse oocyte requires an equilibrium between cyclin B synthesis and degradation. Dev. Biol., 232, 400-413.
7) Naito, K., Hawkins, C., Yamashita, M., Nagahama, Y., Aoki, F., Kohmoto, K., Toyoda, Y. and Moor, R.M. (1995): Association of $\mathrm{p} 34 \mathrm{cdc} 2$ and cyclin B 1 during meiotic maturation in porcine oocytes. Dev. Biol., 168, 627-634.

8) Kalous, J., Kubelka, M., Rimkevicova, Z., Guerrier, P. and Motlik, J. (1993): Okadaic acid accelerates germinal vesicle breakdown and overcomes cycloheximide- and 6dimethylaminopurine block in cattle and pig oocytes. Dev. Biol., 157, 448-454.

9) Aquino, F.P., Naito, K., Cruz, L.C., Sato, E. and Toyoda, Y. (1995): Effects of vanadate on meiotic maturation of porcine oocytes in vitro. J. Reprod. Dev., 41, 271-276.

10) Naito, K. and Toyoda, Y. (1991): Fluctuation of histone H1 kinase activity during meiotic maturation in porcine oocytes. J. Reprod. Fertil., 93, 467-473.

11) Inoue, M., Naito, K., Aoki, F., Toyoda, Y. and Sato, E. (1995): Activation of mitogen- activated protein kinase during meiotic maturation in porcine oocytes. Zygote, 3, 265-271.

12) Ohashi, S., Naito, K., Sugiura, K., Iwamori, N., Goto, S., Naruoka, H. and Tojo, H. (2003): Analyses of mitogenactivated protein kinase function in the maturation of porcine oocytes. Biol. Reprod., 68, 604-609.

13) Katsu, Y., Yamashita, M., Kajiura, H. and Nagahama, Y. (1993): Behavior of the components of maturationpromoting factor, cdc2 kinase and cyclin B, during oocyte maturation of goldfish. Dev. Biol., 160, 99-107.

14) Kobayashi, H., Minshull, J., Ford, C., Golsteyn, R., Poon, R. and Hunt, T. (1991): On the synthesis and destruction of A- and B-type cyclins during oogenesis and meiotic maturation in Xenopus laevis. J. Cell Biol., 114, 755-765.

15) Winston, N.J. (1997): Stability of cyclin B protein during meiotic maturation and the first mitotic cell division in mouse oocytes. Biol. Cell, 89, 211-219.

16) Gautier, J. and Maller, J.L. (1991): Cyclin B in Xenopus oocytes: implications for the mechanism of pre-MPF activation. EMBO J., 10, 177-182.

17) Gallant, P. and Nigg, E.A. (1992): Cyclin B2 undergoes cell cycle-dependent nuclear translocation and, when expressed as a non-destructible mutant, causes mitotic arrest in HeLa cells. J. Cell Biol., 117, 213-224.

18) Kishimoto, T. and Okumura, E. (1997): In vivo regulation of the entry into M-phase: initial activation and nuclear translocation of cyclin B/Cdc2. Prog. Cell Cycle Res., 3, 241-249.

19) Hagting, A., Karlsson, C., Clute, P., Jackman, M. and Pines, J. (1998): MPF localization is controlled by nuclear export. EMBO J., 17, 4127-4138.

20) Ookata, K., Hisanaga, S., Okanio, T., Tachibana, K. and Kishimoto, T. (1992): Relocation and distinct subcellular localization of p34cdc2-cyclinB complex at meiosis reinitiation in starfish oocytes. EMBO J., 11, 1763-1772.

21) Goto, S., Naito, K., Sugiura, K. and Tojo, H. (2001): Localization of cyclin B and MAP kinase around chromosomes of cumulus nuclei transferred into porcine oocytes. J. Mamm. Ova Res., 18, 33-38.

22) Goto, S., Naito, K., Ohashi, S., Sugiura, K., Naruoka, H., 
Iwamori, N. and Tojo, H. (2002): Effects of spindle removal on MPF and MAP kinase activities in porcine matured oocytes. Mol. Reprod. Dev., 63, 388-393.

23) Kotani, T., Yoshida, N., Mita, K. and Yamashita, M. (2001): Requirement of cyclin B2, but not cyclin B1, for bipolar spindle formation in frog (Rana japonica) oocytes. Mol. Reprod. Dev., 59, 199-208.

24) Casas, E., Betancourt, M., Bonilla, E., Duculomb, Y., Zayas, H. and Trejo, R. (1999): Changes in cyclin B localisation during pig oocyte in vitro maturation. Zygote, 7, 21-26.

25) Pines, J. and Hunter, T. (1991): Human cyclins A and B1 are differentially located in the cell and undergo cell cycledependent nuclear transport. J. Cell Biol., 115, 1-17.

26) Pockwinse, S.M., Krockmalnic, G., Doxsey, S.J., Nickerson, J., Lian, J.B., van Wijnene, A.J., Stein, J.L., Stein, G.S. and Penman, S. (1997): Cell cycle independent interaction of $\mathrm{CDC} 2$ with the centrosome, which is associated with the nuclear matrix-intermediate filament scaffold. Proc. Natl. Acad. Sci. USA, 94, 3022-3027.

27) Kuroda, T., Naito, K., Sugiura, K., Yamashita, M., Takakura, I. and Tojo, H. (2003): Analysis of the roles of cyclin B1 and cyclin B2 in porcine oocyte maturation by inhibiting synthesis with antisense RNA injection. Biol. Reprod., 69, (in press).

28) Brandeis, M., Rosewell, I., Carrington, M., Crompton, T., Jacobs, M.A., Kirk, J., Gannon, J. and Hunt, T. (1998): Cyclin B2-null mice develop normally and are fertile whereas cyclin B1-null mice die in utero. Proc. Natl. Acad. Sci. USA, 95, 4344-4349.

29) Minshull, J., Murray, A., Colman, A. and Hunt, T. (1991): Xenopus oocytematuration does not require new cyclin synthesis. J. Cell Biol., 114, 767-772.

30) Ihara, J., Yoshida, N., Tanaka, T., Mita, K. and Yamashita, M. (1998): Either cyclin B1 or cyclin B2 is necessary and sufficient for inducing germinal vesicle breakdown during frog (Rana japonica) oocyte maturation. Mol. Reprod. Dev., 50, 499-509. 